\title{
HYDRO MAGNETIC ELASTIC FREE CONVECTION OF A CONDUCTING ELASTICO-VSCOUS LIQUID BETWEEN HEATED VERTICAL PLATES
}

\author{
P. Sreehari Reddy ${ }^{1}$ A. S. Nagarajan ${ }^{2}$ and M. Sivaiah ${ }^{1}$ \\ ${ }^{1}$ Department of Mathematics, N.B.K.R.S\&A College, Vidyanagar-524413, (AP), India. \\ ${ }^{2}$ Department of Mathematics, N.B.K.R.I.S.T., Vidyanagar-524413, (A.P), India. \\ Email: sreeharireddy8969@gmail.com
}

\begin{abstract}
The natural convection flow of a conducting visco-elastic liquid between two heated vertical plates under the influence of a transverse magnetic field has been studied in this paper. Dimensionless equations of the problem have been solved by the method of successive approximation. Numerical solutions for velocity and temperature have been obtained. The results obtained are discussed with the help of graphs. The effect of magnetic parameter $M$, Visco-elastic parameter $R_{C}$ and the product of Prandtl and Eckert numbers [PE] on velocity and temperature fields are investigated.
\end{abstract}

Key words: Visco-elastic liquid, viscous dissipation, vertical plates, convection, successive approximation.

\section{NOMENCLATURE}

$\begin{array}{llll}\frac{D}{D t} & \text { dissipation function material derivative } & T_{I} & \text { uniform temperature } \\ \nabla & \text { Laplace operator } & & \\ d_{j}^{i} & \text { rate of strain tensor } & \bar{p}_{j}^{i} & \text { rate of stress tensor } \\ K & \text { thermal conductivity } & V^{i} & \text { velocity vector } \\ p_{j}^{i} & \text { extra stress tensor } & M & \text { magnetic parameter } \\ T & \text { temperature of the fluid } & P^{l} & \text { isotopic mean pressure } \\ T_{0} & \text { ambient temperature } & R_{C} & \text { specific heat capacity } \\ u & \text { velocity of the fluid } & \beta & \text { visco-elastic parameter } \\ \lambda & \text { elastic parameter of the dimensions of time } & \mu & \text { coefficient of viscosity } \\ \mu_{e} & \text { coefficient of cross viscosity } & \delta_{j}^{i} & \text { Kronecker deltas } \\ v & \text { kinematic viscosity fluid } & \rho_{0} & \text { fluid density }\end{array}$

\section{Introduction}

The problem of fully developed natural convection in a Newtonian fluid, flowing between two heated vertical plates has been solved by Tanner (1962). Soundalgegakar (1974), Mishra and Mishra (1982), studied the Visco- elastic fluid flow problem of a semi- Infinite space of fluid in contact with a plane wall initially at rest, wall being suddenly accelerated to a constant velocity parallel to itself. The unsteady motion of $2^{\text {nd }}$ order fluid between two infinitely extended non-porous plates when the upper plate is moving with uniform velocity and the lower plate is performing linear oscillations in its own plane has been considered by Sacheti and Bhatt (1975), Dutta (1963), Gulati (1966). Vajravelu and Sastri (1978), studied about Free convective heat transfer in a viscous incompressible fluid confined between a long vertical wavy wall and a parallel flat wall. Free convection and mass transfer flow through a porous medium bounded by an infinite vertical porous plate with constant heat flux was considered by Raptis et al. (1982). Soundalgekar and Patial (1982), have investigated unsteady mass transfer flow past a porous plate. Magneto 
hydrodynamic flow of a viscoelastic fluid past an accelerated plate was considered by Singh (1983), Magneto hydrodynamic flow of non-Newtonian visco-elastic fluid through a porous medium near an accelerated plate was considered by Nabil et al. (2003). Samria et al. (2004), studied about Hydro magnetic free- convection laminar flow of an elasto-viscous fluid past an infinite plate. Bhargava et al. (2003) studied about Numerical solution of free convection MHD micropolar fluid between two parallel porous vertical plates. Free convection boundary layer flow of a non-Newtonian fluid along a vertical wavy wall was considered by Kumari et al. (1997). MHD combined convective flow of a dissipative fluid past a semi-infinite vertical plate was investigated by Takhar et al. (2001). Hazeem Attia (2005) studied about unsteady flow of a dusty conducting fluid between parallel porous plates with temperature dependent viscosity. Radiation effects on free convection flow past a semi-infinite vertical plate with mass transfer was considered by Chamka et al. (2001).

Since many electrically conducting liquids are Visco - elastic non- Newtonian character, the aim of this chapter is to investigate the problem of natural convection flow of a conducting Visco-elastic liquid between two heated vertical plates under the influence of a uniform transverse magnetic field. It is assumed that the configuration is infinitely long in the direction of flow; the fluid in the channel is subject to a buoyancy force causing it to rise. Heat is generated within the fluid by viscous dissipation. We have used the method of successive approximation to determine the velocity and temperature fields. The effects of magnetic parameter $\mathrm{M}$, Visco - elastic parameter $\mathrm{R}_{\mathrm{C}}$, and the product of prandtl and Eckert numbers $[\mathrm{PE}]$ on velocity and temperature fields were investigated.

\section{Formulation and solution of the Problem}

A vertical channel is formed by two infinitely wide parallel plates separated by a distance $2 \mathrm{~h}$. The plates are maintained at a uniform Temperature $T_{1}$ which exceeds the ambient temperature $T_{0}$. An elastico viscous liquid rises in the channel driven by buoyancy forces. The constitutive equations of such a liquid, as given by Noll (1960) are

$$
\overline{P_{j}^{i}}+\lambda P_{j}^{i}=2 \mu d_{j}^{i}+4 \mu_{e} d_{\alpha}^{i} d_{j \alpha}
$$

where $P_{j}^{i}$ is given by

$$
S_{j}^{i}=P_{j}^{i}-P^{1} \delta_{j}^{i}
$$

and $d_{j}^{i}$ is given by

$$
2 d_{j}^{i}=V_{, j}^{i}+V_{, i}^{j}
$$

$\mathrm{V}^{\mathrm{i}}$ is the velocity vector, $\delta_{j}^{i}$ is the Kronecker deltas and the term $\bar{p}_{j}^{i}$ appearing in equ. (2.1) represents the rate of stress tensor, which according to Truesdell is given by

$\overline{P_{j}^{i}}=\frac{\partial P_{j}^{i}}{\partial t}+\partial_{j, K}^{i} V^{k}-P_{k}^{i} V_{, j}^{k}-P_{j}^{k} V_{, k}^{i}+P_{j}^{i} V_{, k}^{k}$

The constitutive equation (1) for the elastico - viscous liquid is similar to that suggested by Oldroyd (1950), and has been used by Sharma (1959) \& Bhatnagar (1966).

The basic equations of the problem of the following continuity equation is

$$
V_{, i}^{i}=0
$$

Momentum equations are

$$
\begin{aligned}
& \rho v \frac{\partial u}{\partial x}=-\frac{\partial P^{1}}{\partial x}-\rho g+\frac{\partial P_{x x}}{\partial x}+\frac{\partial P_{x y}}{\partial y} \\
& 0=-\frac{\partial P^{1}}{\partial y}+\frac{\partial P_{x y}}{\partial x}+\frac{\partial P_{y y}}{\partial y}
\end{aligned}
$$


Energy equation is

$$
\rho c \frac{D T}{D t}=K \nabla^{2} T+\phi .
$$

where $\phi=P_{j}^{i} d_{j}^{i}$.

$$
\frac{D}{D t}=\frac{\partial}{\partial t}+V_{k} \frac{\partial}{\partial x_{k}}, \quad \nabla^{2}=\frac{\partial^{2}}{\partial x^{2}}+\frac{\partial^{2}}{\partial y^{2}}
$$

are dissipation function material derivative and Laplace operator respectively. Since the fluid is slightly conducting, the induced magnetic field can be neglected in comparison with the applied magnetic field [sparrow and cess (1962)]. In the absence of any input electric field and on the assumption that the flow is fully developed, the momentum equation in $\mathrm{X}$ and $\mathrm{Y}$ directions are

$$
\begin{aligned}
& v \frac{\partial^{2} u}{\partial y^{2}}+\frac{3 \lambda \mu_{e}}{\rho}\left(\frac{d u}{d y}\right)^{2} \frac{d^{2} u}{d y^{2}}-\frac{1}{\rho} \frac{\partial p^{1}}{\partial x}-g-\sigma \frac{\mu_{e}^{2} H_{0}^{2}}{\rho} u=0 \\
& \frac{\partial P^{1}}{\partial y}=2 \mu_{e} \frac{d x}{d y} \frac{d^{2} u}{d y^{2}}
\end{aligned}
$$

where $u$ and $v$ are the $\mathrm{X}$ - velocity and kinematic viscosity of the fluid respectively The equation (2.9) on integration gives the pressure distribution in the form

$$
P^{1}=\mu_{e}\left(\frac{d x}{d y}\right)^{2}+\phi(x)
$$

If the liquid is non- Newtonian and inelastic, the Eq. (2.8) shows that, the velocity distribution is the same as that in the Newtonian fluids with the same Kinematic viscosity v. But Equation (2.9) shows that, the cross-viscosity exhibits itself through the increasing pressure at each point of the fluid flow, which will not be considered further. In this chapter the above two important conclusions were previously obtained by Bhatnagar [1966] in a more general way.

In fully developed flow the pressure distribution must be hydrostatic and hence $\quad \frac{\partial P^{1}}{\partial x}=-\rho_{0} g$

Inserting Eq. (2.11) in Eq. (2.8) we obtain,

$$
v \frac{\partial u}{\partial y^{2}}+3 \lambda \frac{\mu_{e}}{\rho}\left(\frac{d u}{d y}\right)^{2}+\beta g\left(T-T_{0}\right)-M u=0
$$

where $\beta$ is defined by

$$
\beta=-\frac{1}{\rho}\left(\frac{\partial \rho}{\partial T}\right) p^{1} \text { and } \mathrm{M}=\sigma \frac{\mu_{e}{ }^{2} H_{0}{ }^{2}}{\rho}
$$

Eq. (2.5) valid for moderate differences between $T_{0}$ and $T_{1}$, is the desired momentum relationship. The energy equation for this flow is

$$
K \frac{d^{2} T}{d y^{2}}+\mu\left(\frac{d u}{d y}\right)^{2}+\lambda \mu_{e}\left(\frac{d u}{d y}\right)^{4}=0
$$

Using the following substitutions

Hydro Magnetic Elastic Free Convection Of A Conducting Elastico-Viscous Liquid Between Heated Vertical Plates 


$$
\bar{u}=\frac{v u}{\beta g h^{2}\left(T_{1}-T_{0}\right)}, r=\frac{y}{h}, \theta=\frac{T-T_{0}}{T_{1}-T_{0}}
$$

in Eqs. (2.12) and (2.14) we obtain the dimensionless equations,

$$
\begin{aligned}
& \frac{d^{2} \bar{u}}{d r^{2}}+\frac{3 R_{c} N}{P E}\left(\frac{d \bar{u}}{d r}\right)^{2}+\frac{d^{2} \bar{u}}{d r^{2}}+\theta+M u=0 \\
& \frac{d^{2} \theta}{d r^{2}}+N\left(\frac{d \bar{u}}{d r}\right)^{2}+\frac{R_{c} N^{2}}{P E}\left(\frac{d \bar{u}}{d r}\right)^{4}=0
\end{aligned}
$$

where $\quad \mathrm{R}_{\mathrm{C}}=\frac{\lambda \mu_{e^{v^{2}}}}{\mu h^{4}}, P=\frac{\mu_{e}}{K}, \mathrm{~F}=\frac{v^{2}}{C h^{2}\left(T_{1}-T_{0}\right)}, N=\frac{\rho \beta^{2} g^{2} h^{4}\left(T_{1}-T_{0}\right)}{k v}$

Equations (2.16) and (2.17) contain two unknowns $\bar{u}$ and $\theta$ and must be solved simultaneously to yield the desired velocity and temperature profiles.

The non-dimensional boundary conditions are,

$$
\begin{aligned}
& \bar{u}( \pm 1)=0 \\
& \theta( \pm 1)=0
\end{aligned}
$$

The non linear differential Equations (2.16) and (2.17) are difficult to solve. We have used the method of successive approximation to determine the velocity and temperature fields.

Assuming the non-dimensional numbers measuring the buoyancy force $\mathrm{N}<<1$ (which is a realistic assumption) we expand $\bar{u}$ and $\theta$ in the ascending powers of $\mathrm{N}$ as follows.

$$
\begin{aligned}
& \bar{u}=u_{0}+N u_{1}+N^{2} u_{2}+\ldots \ldots \\
& \theta=\theta_{0}+N \theta_{1}+N^{2} \theta_{2}+\ldots \ldots
\end{aligned}
$$

Inserting Eqs.( 2.19 ) and ( 2.20 ) into Eqs. ( 2.16 ) and ( 2.17 ) and equating coefficients of temperature upto $\mathrm{N}^{2}$ equal to zero we have the following set of ordinary differential equations

$$
\begin{aligned}
& u_{0}^{11}+\theta_{1}-M u_{0}=0 \\
& u_{1}^{11}+\frac{3 R_{c}}{P E}\left(u_{0}^{2} u_{0}^{11}\right)+\theta_{1}-M u_{1}=0 \\
& u_{2}^{11}+\frac{3 R_{c}}{P E}\left(u_{0}^{2} u_{1}^{11}+2 u_{0}^{1} u_{1}^{1} u_{0}^{11}\right)+\theta_{2}-M u_{2}=0 \\
& \theta_{1}^{11}=0 \\
& \theta_{1}^{11}+u_{0}^{1^{2}}=0 \\
& \theta_{2}^{11}+2 u_{0}^{1} u_{1}^{1}+\frac{R_{c}}{P E} u_{0}^{14}=0
\end{aligned}
$$

where the primes in the above equations denote differentiation w.r. to r. The boundary conditions are

$$
\begin{aligned}
& \mathrm{u}_{\mathrm{i}}=0 \quad \text { at } \quad \mathrm{r}= \pm 1, \mathrm{i}=0,1,2 \\
& \theta_{0}=1 \quad \text { at } \quad \mathrm{r}= \pm 1 \\
& \theta_{\mathrm{i}}=0 \quad \text { at } \mathrm{r}= \pm 1, \mathrm{i}=1,2
\end{aligned}
$$

On solving Equations (2.21) to (2.26) with the boundary conditions (2.27) by using method of successive approximations we obtain, 


$$
\begin{aligned}
& \theta_{0}=1 \\
& \theta_{1}=\frac{1}{8 M^{2} \operatorname{Cosh}^{2} \sqrt{M}}[\operatorname{Cosh} 2 \sqrt{M}-\operatorname{Cosh} 2 \sqrt{M} r]+\frac{1}{4 M \operatorname{Cosh}^{2} \sqrt{M}}\left(r^{2}-1\right)
\end{aligned}
$$$$
\theta_{2}=\frac{2}{\sqrt{M} \operatorname{Cosh}^{2} \sqrt{M}}\left[\frac{3 R_{c}}{\text { PEMCosh } \sqrt[3]{M}}\left(\frac{\operatorname{Cosh} 3 \sqrt{M}}{4 M}-\frac{\operatorname{Sinh} \sqrt{M}}{\sqrt{M}}\right)-\frac{\operatorname{Cosh} 2 \sqrt{M}}{6 M^{3} \operatorname{Cosh}^{2} \sqrt{M}}-\frac{1}{2 M^{2} \operatorname{Cosh}^{2} \sqrt{M}}\right]
$$$$
\left[\frac{-\operatorname{Cosh} 2 \sqrt{M} r}{8 M}+\frac{r^{2}}{4}+\frac{\operatorname{Cosh} 2 \sqrt{M}}{8 M}-\frac{1}{4}\right]+\frac{18 R_{c}}{P E M{ }^{\frac{3}{2}} \operatorname{Cosh}^{4} \sqrt{M}}\left[\left\{\frac{\operatorname{Cosh} 4 \sqrt{M} r}{32 M}-\frac{\operatorname{Cosh} 2 \sqrt{M} r}{8 M}+\frac{r^{2}}{4 M}\right\}\right.
$$

$\left.\left\{\frac{\operatorname{Cosh} 2 \sqrt{M}}{8 M}-\frac{r \operatorname{Sinh} 2 \sqrt{M} r}{8 M}-\frac{\operatorname{Cosh} 4 \sqrt{M}}{32 M}+\frac{\operatorname{Sinh} 2 \sqrt{M}}{8 M}-\frac{1}{4 \sqrt{M}}\right\}\right]-\frac{1}{6 M^{3} \operatorname{Cosh}^{3} \sqrt{M}}$

$$
\begin{aligned}
& \left\{\frac{\operatorname{Cosh} 3 \sqrt{M} r}{18 M}-\frac{\operatorname{Cosh} \sqrt{M} r}{2 M}-\frac{\operatorname{Cosh} 3 \sqrt{M}}{18 M}+\frac{\operatorname{Cosh} \sqrt{M}}{2 M}\right\}-\frac{1}{M^{\frac{5}{2}} \operatorname{Cosh}^{3} \sqrt{M}} \\
& \left\{\frac{r \operatorname{Sinh} \sqrt{M} r}{M}-\frac{2 \operatorname{Cosh} \sqrt{M} r}{M^{\frac{3}{2}}}-\frac{\operatorname{Sinh} \sqrt{M}}{M}+\frac{2 \operatorname{Cosh} \sqrt{M}}{M^{\frac{3}{2}}}\right\}
\end{aligned}
$$

$u_{0}=\frac{1}{M}\left[1-\frac{\operatorname{Cosh} \sqrt{M} r}{\operatorname{Cosh} \sqrt{M}}\right]$

$$
u_{1}=\frac{-\operatorname{Cosh} \sqrt{M} r}{\operatorname{Cosh} \sqrt{M}}\left[\frac{3 R_{c}}{\text { PEMCosh } \sqrt[3]{M}}\left(\frac{\operatorname{Cosh} 3 \sqrt{M}}{4 M}-\frac{\operatorname{Sinh} \sqrt{M}}{\sqrt{M}}\right)-\frac{\operatorname{Cosh} 2 \sqrt{M}}{6 M^{3} \operatorname{Cosh}^{2} \sqrt{M}}-\frac{1}{2 M^{2} \operatorname{Cosh}^{2} \sqrt{M}}\right]+
$$$$
\frac{3 R_{c}}{\text { PEMCosh } \sqrt[3]{M}}\left[\frac{\operatorname{Cosh} 3 \sqrt{M} r}{4 M}-\frac{r \operatorname{Sinh} \sqrt{M} r}{\sqrt{M}}\right]-\frac{\operatorname{Cosh} 2 \sqrt{M} r}{6 M^{3} \operatorname{Cosh}^{2} M}-\frac{r^{2}}{4 M^{2} \operatorname{Cosh}^{2} \sqrt{M}}-\frac{1}{4 M^{2} \operatorname{Cosh}^{2} \sqrt{M}} \text {. }
$$

$$
\begin{aligned}
& u_{2}=\frac{-\operatorname{Cosh} \sqrt{M} r}{\operatorname{Cosh} \sqrt{M}}\left[\left\{a_{1} a_{2} b_{1}-a_{3}\left(a_{4} b_{2}-a_{5} b_{3}-b_{4}\right)+a_{6} b_{5}+a_{7} b_{6}+a_{8} a_{9} b_{7}+a_{10}\left(b_{8}-b_{9}\right)+a_{11} b_{10}\right\}+\right. \\
& \left.\left\{a_{12} b_{11}-a_{13} b_{12}-a_{14} b_{13}+a_{15} b_{14}-a_{16} b_{15}\right\}\right]+a_{1} a_{2}\left\{\frac{-1}{\operatorname{Cosh}^{3} \sqrt{M}}\left(\frac{\operatorname{Cosh} 3 \sqrt{M} r}{32 M}-\frac{r \operatorname{Sinh} \sqrt{M} r}{\sqrt{M}}\right)\right\}-
\end{aligned}
$$$$
a_{3}\left\{\begin{array}{l}
a_{4}\left(\frac{\operatorname{Cosh} 5 \sqrt{M} r}{12 M}-\frac{3 \operatorname{Cosh} 3 \sqrt{M} r}{2 M}+\frac{r \operatorname{Sinh} \sqrt{M} r}{\sqrt{M}}\right)-a_{5}\left(\frac{r \operatorname{Sinh} 3 \sqrt{M} r}{32 M}-\frac{3 \operatorname{Cosh} 3 \sqrt{M} r}{128 M^{\frac{3}{2}}}\right) \\
\frac{r \operatorname{Sinh} \sqrt{M} r}{2 M^{\frac{3}{2}} \operatorname{Cosh}^{2} \sqrt{M}}-\frac{r^{2} \operatorname{Cosh} \sqrt{M} r}{2 M^{\frac{3}{2}} \operatorname{Cosh}^{2} \sqrt{M}} .
\end{array}\right\}+
$$

$a_{6}\left(\frac{\operatorname{Cosh} 4 \sqrt{M} r}{60 M}-\frac{\operatorname{Cosh} 2 \sqrt{M} r}{6 M}-\frac{1}{4 M}\right)+a_{7}\left(\frac{\operatorname{Cosh} 2 \sqrt{M} r}{6 M}+\frac{1}{2 M}\right)+$

$a_{8}\left[a_{9}\left(\frac{\operatorname{Cosh} 3 \sqrt{M r}}{32 M}-\frac{r \operatorname{Sinh} \sqrt{M} r}{\sqrt{M}}\right)\right]+a_{10}\left[\left(\frac{\operatorname{Cosh} 5 \sqrt{M r}}{96 M^{\frac{3}{2}}}-\frac{3 r \operatorname{Sinh} \sqrt{M} r}{32 M}-\frac{\operatorname{Cosh} 3 \sqrt{M} r}{32 M^{\frac{3}{2}}}+\right.\right.$

$\left.\left.\frac{r^{2} \operatorname{Cosh} \sqrt{M} r}{16 \sqrt{M}}-\frac{r \operatorname{Sinh} \sqrt{M} r}{M}-\frac{r \operatorname{Sinh} 3 \sqrt{M} r}{32 M}-\frac{3 \operatorname{Cosh} 3 \sqrt{M} r}{128 M^{\frac{3}{2}}}-\frac{\operatorname{Sinh} \sqrt{M} r}{16} \frac{r}{M}\right) \frac{r}{r}\right]+$ 
$a_{11}\left(\frac{\operatorname{Cosh} 4 \sqrt{M} r}{60 M}+\frac{1}{4 M}\right)+a_{12}\left(\frac{r \operatorname{Sinh} 2 \sqrt{M} r}{6 M}-\frac{2 \operatorname{Cosh} 2 \sqrt{M} r}{18 M^{\frac{3}{2}}}\right)$

$-a_{13}\left(\frac{-\operatorname{Cosh} 2 \sqrt{M} r}{24 M^{2}}-\frac{r^{2}}{4 M}-\frac{1}{2 M^{2}}-\frac{\operatorname{Cosh} 2 \sqrt{M}}{8 M^{2}}+\frac{1}{4 M}\right)-a_{14}\left(\frac{\operatorname{Cosh} 4 \sqrt{M} r}{480 M^{2}}-\frac{\operatorname{Cosh} 2 \sqrt{M} r}{24 M^{2}}-\frac{1}{2 M^{\frac{3}{2}}}-\right.$

$\left.\frac{2 r \operatorname{Sinh} 2 \sqrt{M} r}{3 M}+\frac{8 \operatorname{Cosh} 2 \sqrt{M} r}{9 M^{\frac{3}{2}}}-\frac{r^{2}}{4 M^{\frac{3}{2}}}+\frac{\operatorname{Cosh} 4 \sqrt{M}}{32 M^{2}}-\frac{\operatorname{Cosh} 2 \sqrt{M}}{8 M^{2}}-\frac{\operatorname{Sinh} 2 \sqrt{M}}{8 M^{2}}+\frac{1}{4 M^{\frac{3}{2}}}\right)+$

$a_{15}\left(\frac{\operatorname{Cosh} 3 \sqrt{M} r}{144 M^{2}}-\frac{2 \operatorname{Sinh} \sqrt{M} r}{2 M}+\frac{\operatorname{Cosh} 2 \sqrt{M}}{18 M^{2}}-\frac{\operatorname{Cosh} \sqrt{M}}{24 M^{2}}\right)-a_{16}\left(\frac{r^{2} \operatorname{Cosh} \sqrt{M} r}{4 M^{\frac{3}{2}}}-\frac{r \operatorname{Sinh} \sqrt{M} r}{4 M^{2}}-\right.$

$\frac{2 r \operatorname{Sinh} \sqrt{M} r}{M^{\frac{3}{2}}}+\frac{\operatorname{Sinh} \sqrt{M}}{M^{2}}-\frac{2 \operatorname{Cosh} \sqrt{M}}{M^{\frac{5}{2}}}$.

Where,

$$
\begin{aligned}
& a_{1}=\frac{-3 R_{c}}{P E} ; a_{2}=\frac{3 R_{c}}{P E M \operatorname{Cosh}^{3} \sqrt{M}}\left\{\frac{\operatorname{Cosh} 3 \sqrt{M}}{4 M}-\frac{\operatorname{Sinh} \sqrt{M}}{\sqrt{M}}-\frac{\operatorname{Cosh} 2 \sqrt{M}}{6 M^{3} \operatorname{Cosh}^{2} \sqrt{M}}-\frac{1}{2 M^{2} \operatorname{Cosh}^{2} \sqrt{M}}\right\} \\
& a_{3}=\frac{9 R_{c}^{2}}{P^{2} E^{2} M \operatorname{Cosh}^{3} \sqrt{M}} ; a_{4}=\frac{9}{32 M \operatorname{Cosh}^{2} \sqrt{M}} ; a_{5}=\frac{1}{\sqrt{M} \operatorname{Cosh}^{2} \sqrt{M}} ; a_{6}=\frac{3 R_{c}}{P E\left(6 M^{3}\right) \operatorname{Cosh}^{4} \sqrt{M}} \\
& a_{7}=\frac{3 R_{c}}{P E\left(2 M^{3}\right) \operatorname{Cosh}^{3} \sqrt{M}} ; a_{8}=\frac{6 R_{c}}{P E M \operatorname{Cosh}^{3} \sqrt{M}} \\
& a_{9}=\frac{3 R_{c}}{P E M \operatorname{Cosh}^{3} \sqrt{M}}\left(\frac{\operatorname{Cosh} 3 \sqrt{M}}{4 M}-\frac{\operatorname{Sinh} \sqrt{M}}{\sqrt{M}}\right)-\frac{\operatorname{Cosh} 2 \sqrt{M}}{6 M^{3} \operatorname{Cosh}^{2} \sqrt{M}}-\frac{1}{2 M^{2} \operatorname{Cosh}^{2} \sqrt{M}} \\
& a_{10}=\frac{9 R_{c}^{2}}{P^{2} E^{2} M^{\frac{3}{2}} \operatorname{Cosh}^{5} \sqrt{M}} ; a_{11}=\frac{6 R_{c}}{P E\left(12 M^{3}\right) \operatorname{Cosh}^{4} \sqrt{M}} ; a_{12}=\frac{12 R_{c}}{P E M^{\frac{5}{2}} \operatorname{Cosh}^{4} \sqrt{M}} \\
& a_{13}=\frac{2}{\sqrt{M} \operatorname{Cosh}^{2} \sqrt{M}}\left[\left(\frac{3 R_{c}}{P E M \operatorname{Cosh}^{3} \sqrt{M}}-\frac{\operatorname{Sinh} \sqrt{M}}{\sqrt{M}}\right)-\frac{\operatorname{Cosh}^{2} \sqrt{M}}{6 M^{3} \operatorname{Cosh}^{2} \sqrt{M}}-\frac{1}{2 M^{2} \operatorname{Cosh}^{2} \sqrt{M}}\right] \\
& a_{14}=\frac{18 R_{c}}{\operatorname{PEM}^{\frac{3}{2}} \operatorname{Cosh}^{4} \sqrt{M}} ; a_{15}=\frac{1}{6 M^{3} \operatorname{Cosh}^{3} \sqrt{M}} ; a_{16}=\frac{1}{M^{\frac{5}{2}} \operatorname{Cosh}^{3} \sqrt{M}} \\
& b_{1}=\frac{-1}{\operatorname{Cosh}^{3} \sqrt{M}}\left(\frac{\operatorname{Cosh} 3 \sqrt{M}}{32 M}-\frac{\operatorname{Sinh} \sqrt{M}}{\sqrt{M}}\right) ; b_{2}=\frac{\operatorname{Cosh} 5 \sqrt{M}}{12 M}-\frac{\operatorname{Cosh} 3 \sqrt{M}}{2 M}+\frac{\operatorname{Sinh} \sqrt{M}}{\sqrt{M}} \\
& b_{3}=\frac{\operatorname{Sinh} 3 \sqrt{M}}{32 M}-\frac{3 \operatorname{Cosh} 3 \sqrt{M}}{128 M^{\frac{3}{2}}} ; b_{4}=\frac{\operatorname{Sinh} \sqrt{M}}{2 M^{\frac{3}{2}} \operatorname{Cosh}^{2} \sqrt{M}}+\frac{\operatorname{Cosh} \sqrt{M}}{2 M^{\frac{3}{2}} \operatorname{Cosh}^{2} \sqrt{M}} \\
& b_{5}=\frac{\operatorname{Cosh} 4 \sqrt{M}}{60 M}-\frac{\operatorname{Cosh} 2 \sqrt{M}}{6 M}-\frac{1}{4 M} ; b_{6}=\frac{\operatorname{Cosh} 2 \sqrt{M}}{6 M}+\frac{1}{2 M} ; b_{7}=\frac{\operatorname{Cosh} 3 \sqrt{M}}{32 M}-\frac{\operatorname{Sinh} \sqrt{M}}{\sqrt{M}}
\end{aligned}
$$


P.SreehariReddy, A.S.Nagarajan and M.Sivaiah / Journal of Naval Architecture and Marine Engineering 2(2008) 47-56

$$
\begin{aligned}
& b_{8}=\frac{\operatorname{Cosh} 5 \sqrt{M}}{16 M^{\frac{3}{2}}}-\frac{3 \operatorname{Sinh} \sqrt{M}}{32 M} ; b_{9}=\frac{\operatorname{Cosh} 3 \sqrt{M}}{32 M^{\frac{3}{2}}}-\frac{\operatorname{Sinh} \sqrt{M}}{M}+\frac{\operatorname{Sinh} 3 \sqrt{M}}{32 M}-\frac{3 \operatorname{Cosh} 3 \sqrt{M}}{128 M^{\frac{3}{2}}}+\frac{\operatorname{Cosh} \sqrt{M}}{16 \sqrt{M}}-\frac{\operatorname{Sinh} \sqrt{M}}{16 M} \\
& b_{10}=\frac{\operatorname{Cosh} 4 \sqrt{M}}{60 M}+\frac{1}{4 M} ; b_{11}=\frac{\operatorname{Sinh} 2 \sqrt{M}}{6 M}-\frac{2 \operatorname{Cosh} 2 \sqrt{M}}{\frac{3}{2}} ; b_{12}=\frac{-\operatorname{Cosh} 2 \sqrt{M}}{24 M^{2}}-\frac{1}{2 M^{2}}-\frac{\operatorname{Cosh} 2 \sqrt{M}}{8 M^{2}} \\
& b_{13}=\frac{\operatorname{Cosh} 4 \sqrt{M}}{480 M^{2}}-\frac{\operatorname{Cosh} 2 \sqrt{M}}{24 M^{2}}-\frac{2 \operatorname{Sinh} 2 \sqrt{M}}{3 M}+\frac{8 \operatorname{Cosh} 2 \sqrt{M}}{9 M^{\frac{3}{2}}}-\frac{1}{2 M^{\frac{3}{2}}}+\frac{\operatorname{Cosh} 4 \sqrt{M}}{32 M^{2}}- \\
& \frac{\operatorname{Cosh} 2 \sqrt{M}}{8 M^{2}}-\frac{\operatorname{Sinh} 2 \sqrt{M}}{8 M^{2}} \\
& b_{14}=\frac{\operatorname{Cosh} 2 \sqrt{M}}{144 M^{2}}-\frac{\operatorname{Sinh} \sqrt{M}}{2 M}+\frac{\operatorname{Cosh} 3 \sqrt{M}}{18 M^{2}}-\frac{\operatorname{Cosh} \sqrt{M}}{2 M^{2}} ; \\
& b_{15}=\frac{\operatorname{Cosh} \sqrt{M}}{4 M^{\frac{3}{2}}}-\frac{\operatorname{Sinh} \sqrt{M}}{4 M^{2}}-\frac{2 \operatorname{Sinh} \sqrt{M}}{M^{\frac{3}{2}}}+\frac{\operatorname{Sinh} \sqrt{M}}{M^{2}}-\frac{2 \operatorname{Cosh} \sqrt{M}}{M^{\frac{5}{2}}}
\end{aligned}
$$

\section{Results and Discussions.}

The effect of magnetic parameter $M$ on $u_{0}, u_{1}, u_{2}, \theta_{1}$ and $\theta_{2}$ is illustrated in Figures (1) to (5). From Figs. (1) \& (2), it is observed that $\mathrm{u}_{0}, \mathrm{u}_{1}$ decreases as the magnetic parameter $\mathrm{M}$ increases. It is because of that, the application of transverse magnetic field will result a resistive type force, which is known as Lorentz force, which tends to resist the fluid flow and thus reduces velocity. From Fig. (3), it is clear that $u_{2}$ increases as M increases. In Fig. (4), we observe that $\theta_{1}$ decreases as $\mathrm{M}$ increases where as from Fig. (5), it is observed that $\theta_{2}$ increases as $\mathrm{M}$ increases. A close look at Figs. (6) and (7), will reveal the fact that $\mathrm{u}_{1}$ and $\mathrm{u}_{2}$ increases as PE [Product of Prandtle and Eckert numbers] increases. Physically, it is possible because fluids with high Product of Prandtle and Eckert numbers have low viscosity and hence move faster. Fig. (8) shows that $\theta_{2}$ increases as PE increases. This is due to the fact that
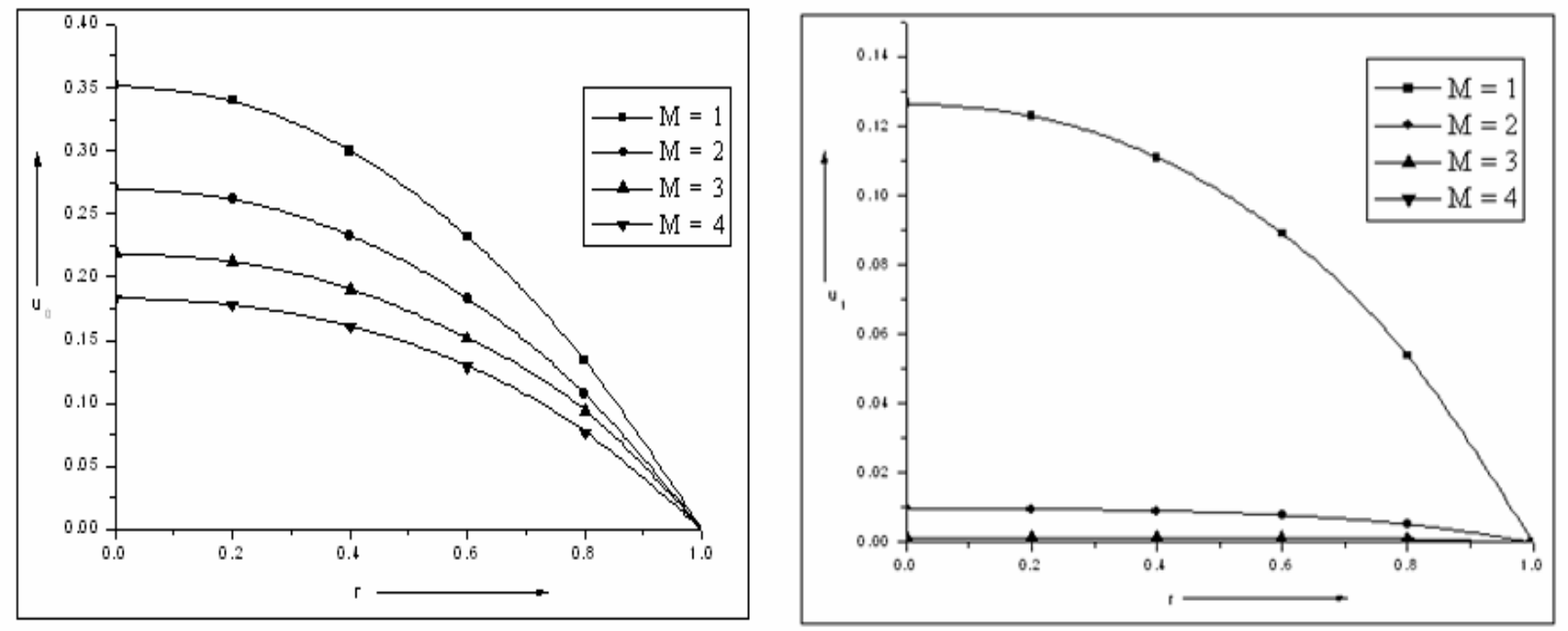

Fig. 1: Effect of $M$ on $u_{0}$

Fig. 2: Effect of $M$ on $u_{1}$ 
P.SreehariReddy, A.S.Nagarajan and M.Sivaiah/ Journal of Naval Architecture and Marine Engineering 2(2008) 47-56

thermal conductivity of fluid increases with increasing PE, resulting an increase in thermal boundary layer thickness. From fig. (9) and (10), we observe that both $u_{1}$ and $u_{2}$ decreases as the viscoelastic parameter $R_{C}$ increases. Fig. (11), shows that $\theta_{2}$ decreases as the viscoelastic parameter $R_{C}$ increases.

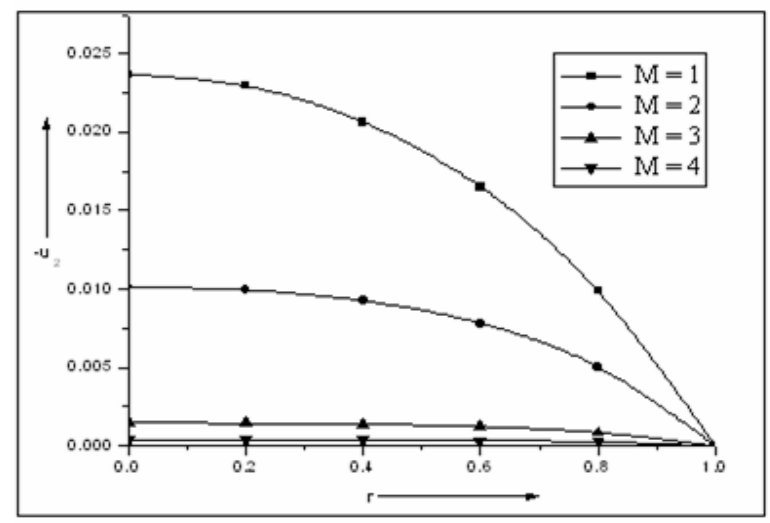

Fig. 3: Effect of $M$ on $u_{2}$

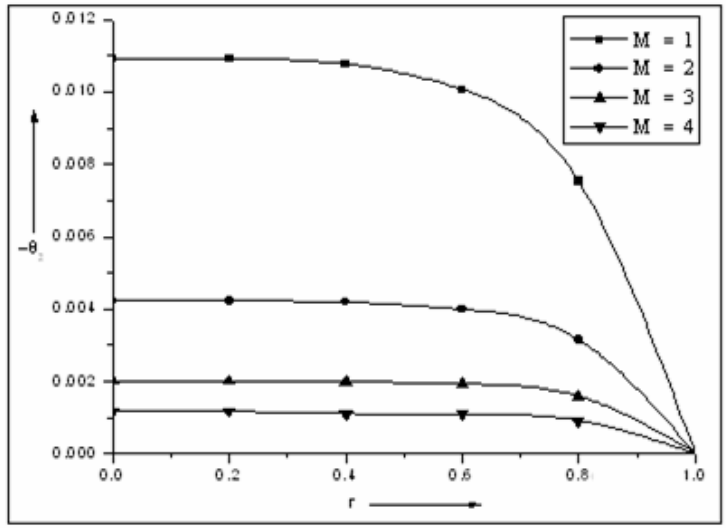

Fig. 5: Effect of $M$ on $\theta_{2}$

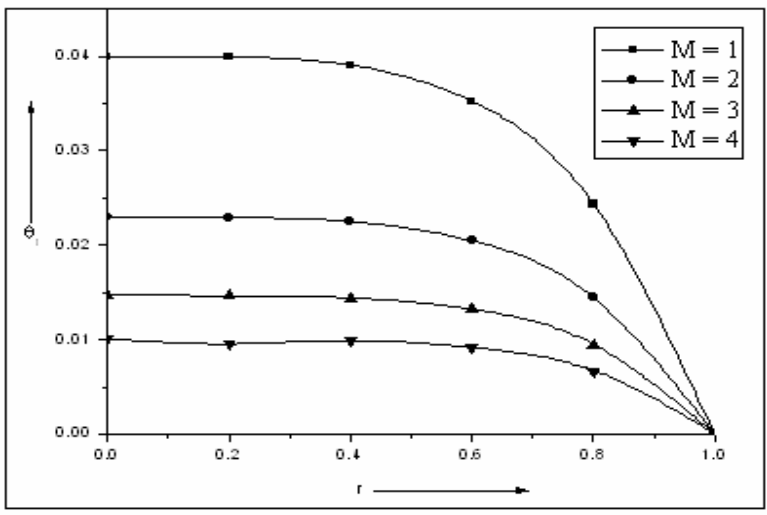

Fig. 4: Effect of $M$ on $\theta_{1}$

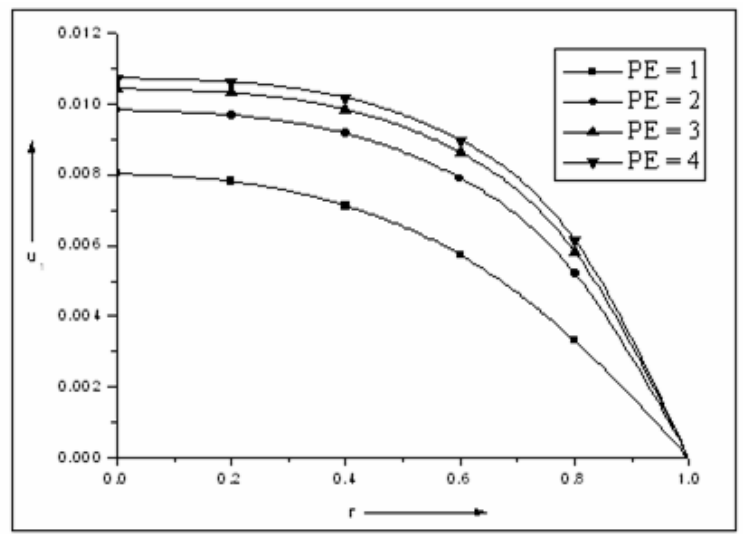

Fig. 6: Effect of $P E$ on $u_{1}$

\section{Conclusions.}

The aim of this paper is to investigate the problem of natural convection flow of a conducting visco-elastic liquid between two heated vertical plates under the influence of a uniform transverse magnetic field. Results found from this study can be summarized as follows:

1) With the increase in visco-elastic parameter $R_{c}$ the velocity and temperature decreases.

2) The velocity decreases with the increase of magnetic parameter $M$.

3) The velocity and temperature increases with the increase of product of Prandtl and Eckert number [PE]. 
P.SreehariReddy, A.S.Nagarajan and M.Sivaiah/ Journal of Naval Architecture and Marine Engineering 2(2008) 47-56
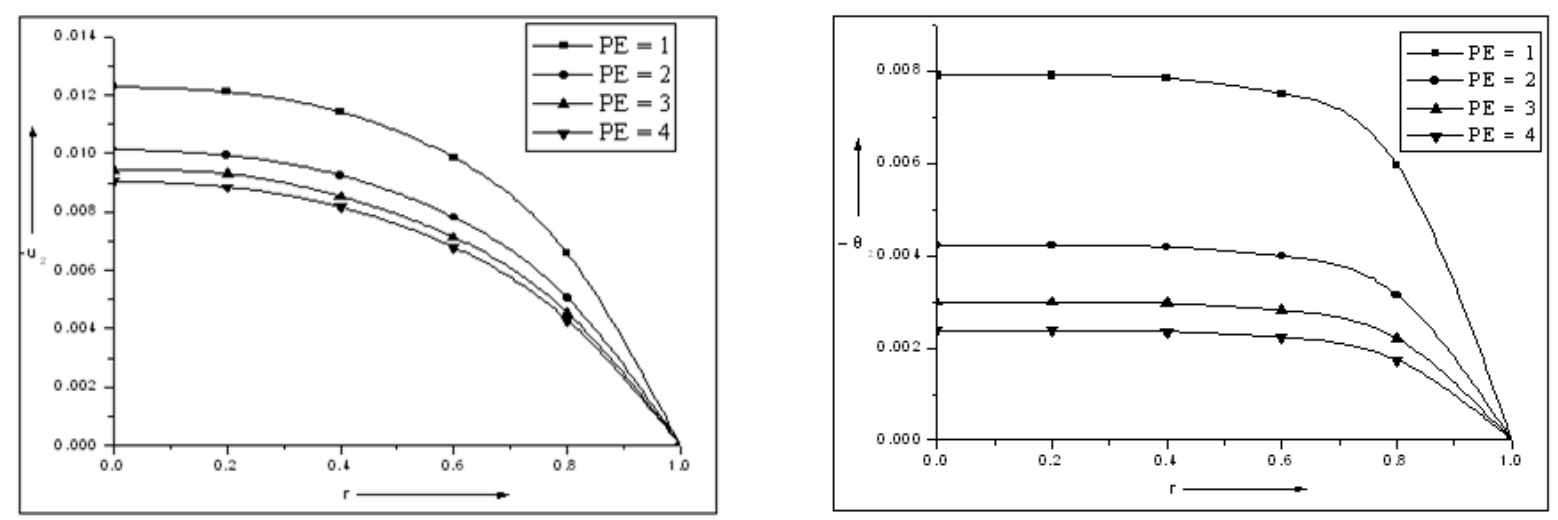

Fig. 7: Effect of $P E$ on $u_{2}$

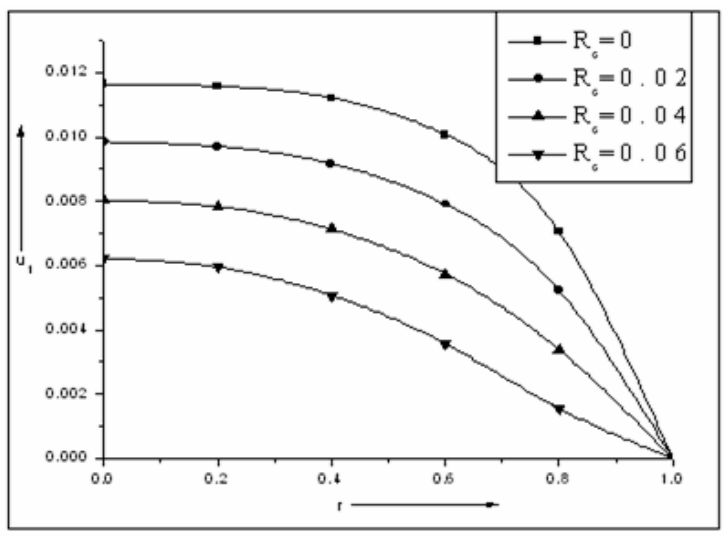

Fig. 8: Effect of $P E$ on $\theta_{2}$

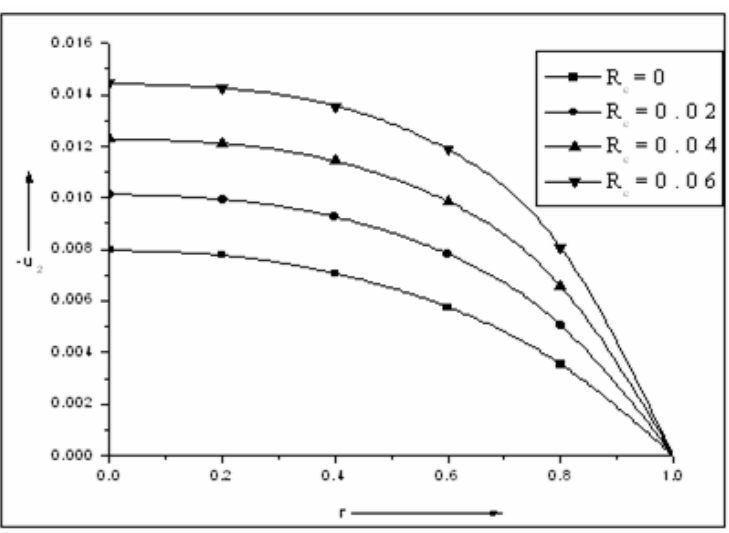

Fig. 9: Effect of $R_{c}$ on $u_{1}$

Fig. 10: Effect of $R_{c}$ on $u_{2}$

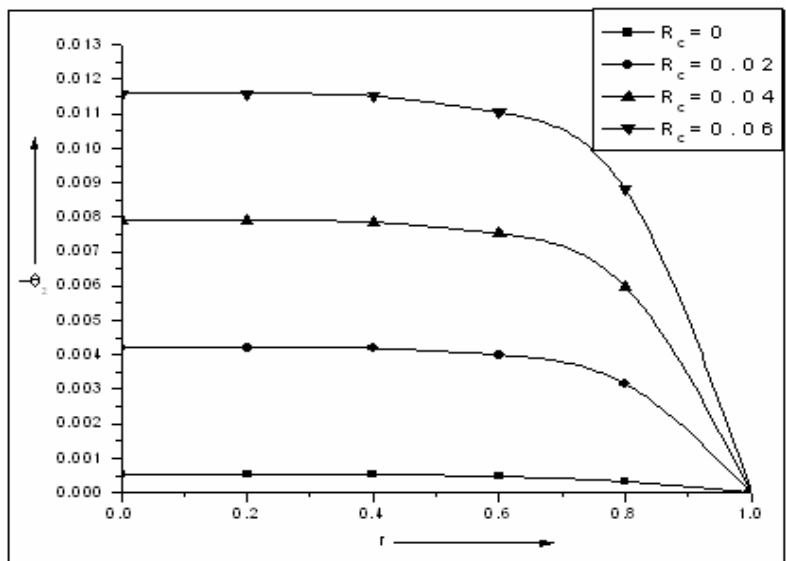

Fig. 11: Effect of $R_{c}$ on $\theta_{2}$ 


\section{References}

Bhargava, R., Kumar, L. and Takhar, H.S. (2003): Numerical Solution Of Free Convection MHD Micropolar Fluid Between Two Parallel Porous Vertical Plates, Int. J. Eng. Sci. 41, 123.

doi:10.1016/S0020-7225(02)00157-X

Bhatnagar, P.L. (1966): Laminar Flow Of An Elastico-Viscous Fluid Between Two Parallel Walls With Heat Transfer, ZAMP 17, 646. doi:10.1007/BF01597248

Chamkha, A.J., Takhar, H.S. and Soundalgekar, V.M. (2001): Radiation Effects On Free Convection Flow Past A Semi-Infinite Vertical Plate With Mass Transfer, Chemical Engineering Journal, 84, 335.

doi:10.1016/S1385-8947(00)00378-8

Dutta, S.K. (1963): Flow Formation In Coutte Motion Of An Elastico-Viscous Maxwell Fluid In The Presence Of A Transverse Magnetic Field, J.Phys.Soc., Japan 18, 1667. doi:10.1143/JPSJ.18.1667

Gulati, S.P. (1966): The Flow Of An Elastico-Viscous Liquid Between Two Electrically Non-Conducting Infinite Flat Plates Under A Uniform Transverse Magnetic Field, J.Phys.Soc., Japan 21, 1411. doi:10.1143/JPSJ.21.1411 Hazem Attia, A. (2005): Unsteady Flow Of A Dusty Conducting Fluid Between Parallel Porous Plates With Temperature Dependent Viscosity, Turk J. Phys. 29, 257.

Kumari, M., Pop, I. and Takhar, H.S. (1997): Free Convection Boundary Layer Flow Of A Non-Newtonian Fluid Along A Vertical Wavy Wall, Int.J. Heat and Fluid Flow, 18, 525-632. doi:10.1016/S0142-727X(97)00024-6 Mishra, S.P. and Mishra, K.C. (1982): Visco-Elastic Flow Due To A Plate Which Starts Oscillating In The Presence Of A Parallel Stationary Plate, Rheol, Acta 21, 25.

Nabil, T. M. Eldabe, Galal M.Moatimid, and Hoda Sm Ali (2003): Magneto Hydrodynamic Flow Of NonNewtonian Visco-Elastic Fluid Through A Porous Medium Near An Accelerated Plate, Can.j.Phys/Rev.can.phys.81 (11):1249-1269.

Noll, W.J. and Truesdell, C. (1960): The Non-Linear Fluid Theories, Hand Buch Der Physic, III, Springer link, Berlin.

Oldroyd, J. G. (1950): On The Formation Of The Rheological Equations Of State, Proc. R. Soc., 200, 523. doi:10.1098/rspa.1950.0035

Raptis, A, Kafousias, N and Massalas, C. (1982): Free Convection And Mass Transfer Flow Through A Porous Medium Bounded By An Infinite Vertical Porous Plate With Constant Heat Flux, ZAMM 62, 489. doi:10.1002/zamm.19820620911

Sachetic, N.C. and Bhatt, C.S. (1975): The Unsteady Motion Of 2nd Order Fluid Between Two Infinitely Extended Non-Porous Plates, IJPAM, 6, 996.

Samaria, N.K., Reddy, M.U.S., Prasad, R. and Gupta, H.N. (2004): Hydro Magnetic Free- Convection Laminar Flow Of An Elasto-Viscous Fluid Past An Infinite Plate, Springer link Vol.179, No.1.

Sharma, S.K. (1959): Heat Transfer By Laminar Flow Of An Elastico-Viscous Liquid In A Circular Cylinder With Linearly Varying Wall Temperature, ZAMM, 39, 313. doi:10.1002/zamm.19590390709

Singh, A. (1983): Magneto Hydrodynamic Flow Of A Visco-Elastic Fluid Past An Accelerated Plate, Nat.Acad.Sci.Lett.6, 233.

Soundalgekar, V. and Patial, V. (1982): Unsteady Mass Transfer Flow Past A Porous Plate, Pure and Appl.Math 13,399 .

Soundalgekar, V.M. (1974): Stokes Problem For Elastico-Viscous Liquid, Rheol Acta, 13, 177.

doi:10.1007/BF01520872

Sparrow, E. M. and Cess, R. D. (1962): Magnetohydrodynamic Flow And Heat Transfer About A Rotating Disk, Trans ASME, E; J. Appl. Mech. 29, 181.

Takhar, H.S., Soundalgekar, V.M., Deka, R. and Das, U.N. (2001): MHD Combined Convective Flow Of A Dissipative Fluid Past A Semi-Infinite Vertical Plate, J. MHD, Plasmas \& Space Research, 10, 1-14. Tanner, R. (1962): Notes On The Rayleigh Parallel Problem For A Viscoelastic Fluid, I ZAMP, 13, 573. doi:10.1007/BF01595580

Vajravelu, K and Satri, K. (1978): Free Convective Heat Transfer In A Viscous Incompressible Fluid Confined Between A Long Vertical Wavy Wall And A Parallel Flat Wall, J.Fluid Mech.86, 365. doi:10.1017/S0022112078001172 